\title{
A Non-invasive Wireless Monitoring Device for Children and Infants in Pre-hospital and Acute Hospital Environments
}

\author{
Phillip Winderbank-Scott and Payam Barnaghi \\ Department of Electronic and Electrical Engineering \\ Institute for Communication Systems (ICS) \\ University of Surrey, Guildford, United Kingdom
}

\begin{abstract}
This paper describes the design and development of a wireless monitoring system for use within a pediatric environment. The current wired methods used to provide noninvasive sensing are not best suited to their end user, and there is a development need for platform independent data transmission.

The main goal has been to develop a practical and flexible proof-of-concept prototype suitable for the transmission of sensor data. This prototype consists of an Arduino based multi-input sensor system with wireless transmission, and an Android monitoring station with the facility to rebroadcast the collected data via email/web as a data file. This was achieved using commercially available hardware platforms. The software produced for the Android device allows for full control of the functionality provided by the sensor platform developed on the Arduino system, as well as storing the data within a relational database. The data can also be graphically represented in realtime on the Android device.
\end{abstract}

Keywords-Pediatric; monitoring; Arduino; Android; CookingHacks eHealth; Piecewise Aggregate Approximation; Symbolic Aggregate Approximation

\section{INTRODUCTION}

\section{A. Background and Context}

According to data published by the Health and Social Care Information Centre in the UK, pediatric inpatient care accounted for the third highest number of patients at over 1.4 million/year [1]. By designing a system that will enable healthcare providers to remotely monitor various vital statistics, more cost effective solutions are possible, for example reducing the number of outpatient appointments required by monitoring patients at home.

The target market for this device is initially within the pediatric field. However, there is scope for this prototype to provide a more generic solution that could be used within most forms of patient monitoring. A press release by the International Data Corporation (IDC) in 2014 [2], suggests that by 2018 over $70 \%$ of healthcare providers worldwide will have invested in some form of wearable healthcare monitoring system in order to reduce costs and provide a more sustainable solution.

\section{B. Hardware overview}

One of the underlying aims of the project was to use existing embedded hardware technology to enable development of a rapid and cost-effective design. The eHealth shield was chosen as it interfaces to existing sensors and some libraries have been written to enable data access. Whilst there exist many different types of hardware that could have been used for the processor section of the project, use of the eHealth shield precluded this to either an Arduino based system or a Raspberry Pi. Arduino based systems are designed to repetitively carry out sampling tasks and unlike Raspberry Pi, these programs run straight on the processor without having to interface with an operating system (Linux in the case of the Raspberry Pi). Development for an Android system was a much more straight forward decision as its open platform nature allows for a far greater freedom in publishing software.

The system developed for this project is illustrated with the block diagram shown in Fig. 1. The Cooking Hacks e-Health Sensor Shield [3], allows the connection of various body sensors. As the sensor shield is based around an Arduino (also known as the Genuino outside of America) board, and due to the complexity of the project, the chosen processor module was the Arduino Mega 2560 [4]. The obvious choice for a communication method was Bluetooth Low Energy due to its low power consumption. With most mobile devices containing Bluetooth, this provided for a multiplatform solution. The Arduino compatible board used was the Adafruit Bluetooth LE shield [5].

\section{Prototype development}

\section{1) Proof-of-concept prototype}

The initial hardware was capable of retrieving data from sensors and wirelessly transmitting this data to the Android device. The sensor unit was based on the Arduino Mega 2560 board and connection to the eHealth board is via the many $\mathrm{I} / \mathrm{O}$ ports available.

The Bluefruit BLE shield connected to the Arduino board via an SPI interface. This prototype used live data for the temperature sensor, with pseudo values used for other sensors. 


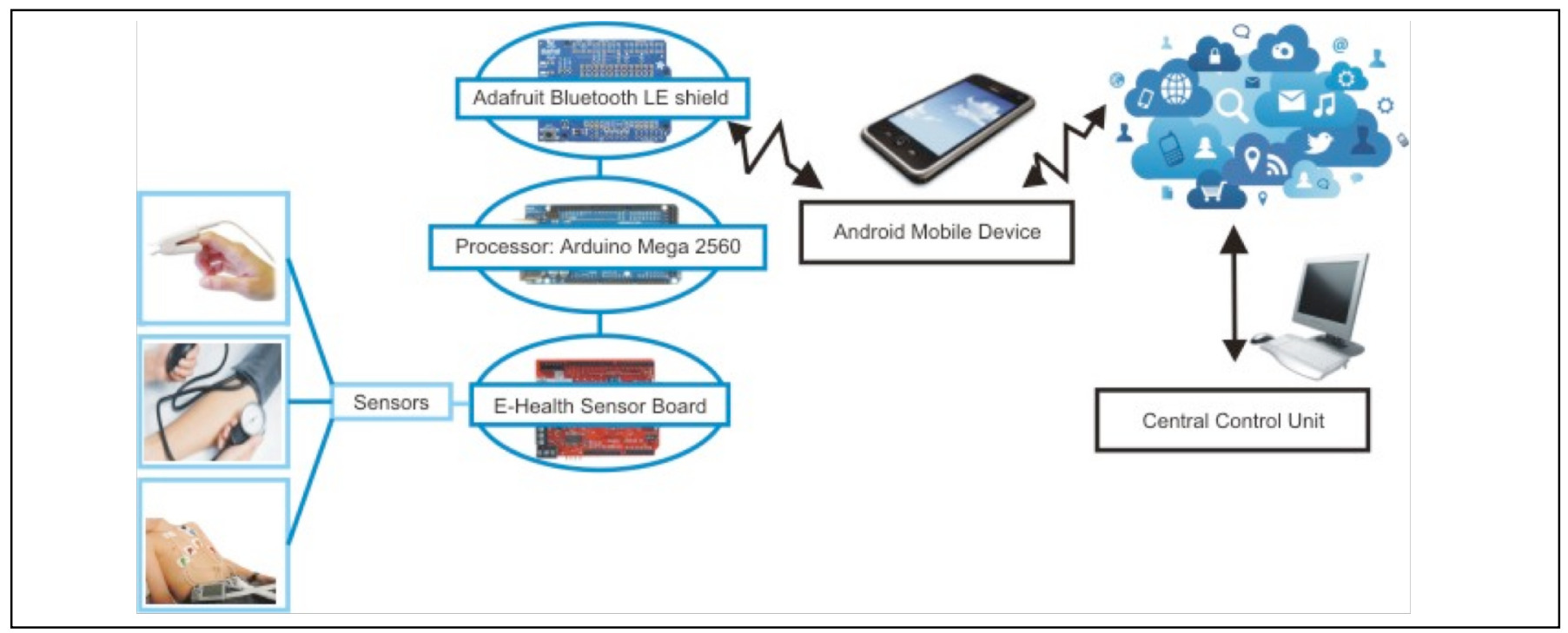

Fig. 1. The overall architechture of the system

The provided sensor interface libraries allowed rapid development of the Arduino software, but there appeared to be interference between the sensors when attempting to sample more than one. The data was transmitted using a hardware UART (serial port). This proved to be very inefficient. In addition, the user could select the type of sensor to be used from a predefined list and this data would then be transmitted. At this stage, the Android device would interpret the data and allow for it to be graphically represented in real time. All of the software developed for this prototype was modular in nature, and this proved beneficial for the changes implemented in the second prototype.

\section{2) GATT Characteristics based prototype}

Learning from the issues raised by the first prototype, a modified design was produced. This used the same hardware as was initially developed, but changed the method for data delivery. This design made use of the GATT services and characteristics available within the BLE framework. By implementing this method of data transmission each item of data could be individually controlled without having any effect on another which allowed for a far more scalable design. Other features developed for this prototype included automatic discovery of the device capabilities and sensor availability, as well as the availability of live data for the SpO2, BPM and ECG sensors.

\section{3) eHealth Monitor with data compression}

Building on the second prototype, additional features were introduced including Piecewise Aggregate Approximation and Symbolic Aggregate Approximation compression for the ECG measurements and Piecewise Aggregate Approximation and Piecewise Linear Approximation for the temperature measurements. The hardware design was also modified to allow for parallel data transmission, thus allowing for a greater data throughput. As a proof of concept model, the maximum data rate for this prototype is currently just over $200 \mathrm{~Hz}$, but by adding additional BLE boards this could be increased to $500 \mathrm{~Hz}$ or beyond.

\section{STATE-OF-THE-ART}

\section{A. Research from an electronics perspective}

One of the first references found to the use of sensing platforms is in a paper presented in 2001 by Dr V Jones et al. [6]. This paper uses the concepts of a VAN (vehicle area network) and a BAN (body area network) to propose the use of a PAN (personal area network). The express use of these networks was to allow for the communication of data from an ambulance to a hospital (VAN), from the paramedic to the hospital (BAN) and then from the home of the patient in an aftercare scenario via the PAN in order to reduce the ongoing cost of patient care.

The EU Mobihealth project investigated the use of mobile sensor networks in healthcare. Mobihealth used a BAN to provide data back to a central location; in this case via 2.5/3G [7].

The Mobihealth project [8] implemented a system that would continue to appear in some form in future papers. This consisted of sensors talking to a mobile base unit (MBU) and data passing from the MBU to a central location. At this time data transmission was via GPRS, and the publication describes issues not only with communication between the sensors and the MBU but also between the MBU and the central location. The issues found between the MBU and the central location was caused by the use of HTTP. As the GPRS link was not stable, using a protocol that required acknowledgement of receipt of data packets caused a backup of the packets.

Lo Benny et al. [9] discuss the use of a Body Sensor Network for cardiac monitoring. They discuss the use of wireless sensors reporting to a body worn Local Processing Unit. This would then process the data and retransmit but no mention is made of development past the demonstration stage. Otto et al. [10] also describe a system for ECG monitoring that uses a PDA and nodes based on TinyOS [11] which is an event based operating system designed for low power nodes and 
sensors. Milenković et al. [12] discuss Wearability, Communication Reliability, Security and Interoperability as the four main considerations for the development of a mobile monitoring system

Abidoye et al. [13] discuss a theoretical three tier system. The first tier consists of collecting data locally onto a Medical Super Sensor. This data is then transferred to the second tier, a PDA, using a technology such as ZigBee or Bluetooth. At this point the data is aggregated and transferred via Wi-Fi or $3 \mathrm{G}$ to the final tier which would allow for the accessing of data onto a medical server or by a doctor/nurse.

Further, Abidoye et al. discuss the benefits of ZigBee versus Bluetooth. The authors argue that as their system is based around their Medical Super Sensor, Bluetooth is not a suitable architecture as it would only allow for seven slave units to connect to the Super Sensor. This is not however the case with newer technologies such as Bluetooth Low Energy.

Finally, suggestions are made as to the usage of such a platform and suggestions are made for heart disease monitoring, asthma monitoring, cancer detection, diabetes as well as in artificial retinas. This further strengthens the case that a suitably developed platform could have wider implications than just the pediatric healthcare market. In a more recent paper by Snehalatha and Shruti [14] a solution was proposed for a system that could be produced using an ATMega chipset with attached sensors, connecting via Bluetooth to a mobile phone. Even here though it would appear that no practical solution was ever developed further than to the block-diagram stage. Interestingly, the applications of a monitoring system are further widened to include sports monitoring.

One of the main proponents of health monitoring is SHIMMER $^{\mathrm{TM}}$ [15] . Founded in 2008, their initial work was based on the TinyOS platform [16]. SHIMMER ${ }^{\mathrm{TM}}$ stands for Sensing Health with Intelligence, Modularity, Mobility and Experimental Reusability. Current work by the company has expanded into the arena of Big Data with one of the latest publications outlining XML communication to a Cloud based system [17].

\section{B. Research from a medical perspective}

Discussion of the use of remote monitoring appears in the literature as far back as 1971 [18]. In this paper, the author describes the use of an FM telemetry device and mentions the advantages of its use in the pediatric environment. More recently, in 1997, a paper was published regarding the use of twenty-four hour monitoring in children [19]. This paper researched the use of a backpack mounted blood pressure monitoring device for 24 hour periods on 3 to 6 year old children. Whilst the data was not then retransmitted, the paper does make mention of some of the advantages of such systems such as a reduction in the stress caused to children in a hospital environment as well as discussing the virtues of a relative high initial investment cost, to a reduction in manpower and ongoing costs. On a negative side, it points out the fact that within one hour of application, seven of the sample sixty-one children had managed to remove the sensing device and I feel this further illustrates the need to provide a well thought out and researched sensor design for use with any final equipment.

A short reference, but relevant to the whole ethos of the project, a paper from 2011 by Salgado et al. [20] positively reflects on, and empirically discusses the advantages of nonhospital based monitoring in the pediatric environment.

Saarel et al. [21] describe the use of a system called CardioNet [22]. This initially consisted of a small three electrode wireless ECG sensor, transmitting to a larger monitor with built in mobile phone unit. The CardioNet unit is now considerably smaller, but the principles behind its design and use remain the same.

The ECG system manufactured by Isansys [23] has been updated since the publication by Saarel et al. and now comprises sensors for all age ranges. These sensors are fully wireless, and various versions are available for measurement of ECG, SpO2, temperature and blood pressure. The data from these is fed back to a local monitor called a Patient Status Engine that can display all information regarding a patient and then retransmit to a central station. The paper also discusses a $\mathrm{SpO} 2$ sensor called Wristox manufactured by Nonin [24]. This comprises a wrist worn system with a finger sensor that allows download of the collected data via USB or Bluetooth. Perhaps unsurprisingly, they note a higher patient compliance with the unobtrusive wireless sensors used in the measurement of the ECG than with the bulky sensor used in the $\mathrm{SpO} 2$ monitor.

\section{Compression techniques}

\section{1) Piecewise Data Compression}

Piecewise data compression describes compression techniques that divide a continuous time stream of data into segments. These segments are then averaged in some manner and this value then replaces the measured values. The main parameter that can be altered in this type of compression is the segment size. The two main disadvantages to a larger segment size are the loss of fine data measurement changes and the size of the processing buffer required. The main advantage however is that by performing this type of compression at the front end of any transmission system, the amount of data transmitted decreases as an inverse proportion to the segment size.

\section{2) Piecewise Linear Approximation}

Piecewise Linear Approximation (PLA) was first discussed in a paper from 1960 by Cameron [25]. This compression technique takes a data stream and reduces segments of the stream to linear representation. The size of these segments can be determined in several ways including Sliding Window, Topdown and Bottom-up. Sliding window compression is more commonly used on real time data whereas top-down and bottom-up tend to be used for stored data sets. This method of data compression represents data trends rather than closely representing the data itself. In this regards, it can be useful for pattern matching algorithms.

\section{3) Piecewise Aggregate Approximation}

Piecewise Aggregate Approximation (PAA) was first discussed in a paper by Keogh et al. [26]. The principle of PAA is to define a segment and then provide each point in the segment with an average value. The segment can be of varying 
length, but with the main purpose of this type of compression being the simplicity and speed of its application, it is more common to use a segment of fixed size.

\section{4) Symbolic Time-series Approximation}

Symbolic Time-series Approximation (STA) is again mainly used to provide a method of pattern recognition. In a paper by Sant'Anna et al. [27] the suggestion is made that there are four main areas of interest in an STA. These are Alphabet size, Information loss, Compression, and Temporal information. The use of a larger alphabet increases the resolution of a signal, but in turn this proportionally increases the amount of data to send. Conversely, by decreasing the alphabet size, the amount of data sent is reduced, but also the contained information can become difficult to interpret. As with all compression techniques, there is a computational overhead to processing the data and for time critical applications, the smaller the alphabet size, the simpler the computation.

\section{5) Symbolic Aggregate Approximation}

Symbolic Aggregate Approximation (SAX) is a form of STA, and was first introduced in a paper by Lin et al. from 2003 [28]. SAX initially uses normalisation to present a common time series. PAA is then used to reduce the number of levels in the time series. In order to convert the reduced time series into a set of equi-probable characters, this new time series is then converted into character representation using approximations based on a Gaussian probability curve. The character breakpoints are applied to the time series according a derived table.

\section{6) ECG specific compression}

Various papers have been written looking at the use of SAX in pattern recognition for ECG signals. Kulahcioglu et al. [29] studied this application and analysed the loss of information from the original time-series due to different sample sizes. They then looked at inclusion of a deliberate anomaly; in this case, an arm moving whilst the ECG reading was taken. Basing their study on the use of both PLA and PAA to provide the initial data reduction and then using extended SAX to symbolise the resulting series, they found limited use for detecting aperiodic anomalies due to similarities to the actual data.

Singh et al. [30] analysed other compression techniques, moving from simple one dimensional to more complex two dimensional systems that are used for image compression. This change was instigated by the realisation that by looking at the signal on a beat level, not only were there sample-to-sample (intra-beat) correlations which could be managed by one dimensional compression techniques, but also there were similarities to the signal on a beat-to-beat (inter-beat) basis that could be further compressed.

\section{DESIGN AND DEVELOPMENT}

The design and development carried out in this project can be divided between hardware and software; and the software development can be further divided into Android development for the user interface and control platform and Arduino development for the sensor platform. Full listings of all of the software developed for this project can be found at https://github.com/UniSurreyIoT/ehealth-monitoring.

\section{A. Hardware}

The aim of this project was to use existing of-the-shelf hardware with a view to future miniaturisation. Working down from the top, 0 shows the eHealth Sensor board, two Adafruit Bluetooth LE boards and the Arduino Mega 2560 processor board. As shown in Fig 2. the pulse oximetry and heart rate sensor and the ECG sensor leads are also attached to the board. The use of Bluetooth LE was considered due to the interoperability of this transmission method with standard phones and computers. In a paper by Gomez et al. [31], the power consumption of BLE device powered by a CR2032 battery was investigated and the conclusions showed that a transmission every $32 \mathrm{~s}$ would lead to a battery life of 12.4 years, dropping to a transmission every $7.5 \mathrm{~ms}$ giving a battery life of 2 days.

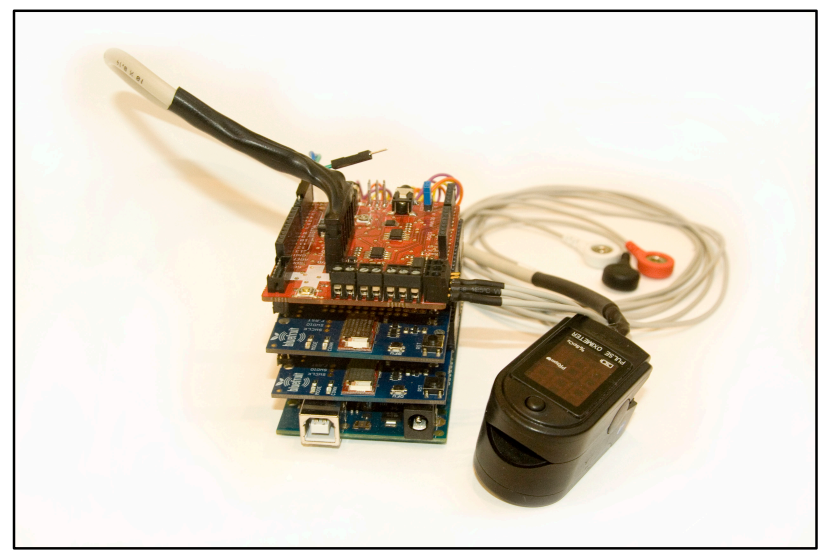

Fig. 2. Thw prototype hardware

\section{B. Software development}

Initial investigations for the design of the software were based around the programs available from the manufacturer of the BLE device. These programs allowed a user to connect to an available BLE device and look at the characteristics in use; in this case, a simple pseudo UART. Making use of this UART facility, data could then be transmitted between the Android device and the Arduino device.

One of the main programming challenges in developing a design making use of strings of data was enabling simple parsing of the data. In order to make the data readily available, the initial design placed the data in a string of comma separated variables, with known locations in the string for each of the sensors and any other data. When additional functions, such as reading available sensors and changing the sampling interval, were added to the program, this also required the use of a header function in the string to differentiate between data strings and setting strings.

Further complications were added by the fact that the UART available was in fact not a true serial link, but was based on two twenty byte GATT characteristics with no flow control. 
The GATT (Generic Attribute Protocol) is a method of defining services and characteristics available on a BLE enabled device. One GATT characteristics was used for transmitted data and the other characteristic was used for received data. No flow control exists in the available libraries provided by the device manufacturer This meant that whilst the majority of the time the required data string was transmitted as required, but, on some occasions either a string was sent before it was fully formed or alternatively on other occasions two strings would be partially concatenated and parts of both strings would form the 20-byte packet. This caused intermittent issues with parsing at the Android device, as each 20-byte packet would be forwarded to the parser when available. These issues were leading to lost data and incorrect data as well as delays in displaying the data.

Following on from this initial prototype, further investigation was carried out into the use of the BLE GATT services and characteristics to overcome these issues. Each GATT service and characteristic consists of several parts including a unique identifier (the UUID) and a payload. Each GATT can be defined by one of five types: Read, Write without Response, Write, Notify and Indicate. By defining a characteristic with a notify type, any change to the characteristics will be transmitted by the remote device and received by the master device in an autonomous fashion. This means that the remote device does not need to be polled for new data and this in turn reduced power consumption.

Several issues were found with the implementation of the GATT services and characteristics between the Android and Arduino systems. These included the use of Little Endian vs Big Endian implantations as well as the BLE library only parsing single byte data. A translation function was written to cope with the first problem and the manufacturer's library was modified to provide a solution to the parsing issue.

Having developed a stable platform, additional features such as Piecewise Aggregate Approximation and Symbolic Aggregate approximation were added to the software.

\section{Additional Harware Development}

After work had been completed on the software compression methods, results showed a minimum reliable sampling period of $10 \mathrm{~ms}(100 \mathrm{~Hz})$. It has been shown in a number of works [32], [33] and [34], that ECG measurements in a pediatric environment should have a minimum sampling frequency of $500 \mathrm{~Hz}$, with the suggestion that in order to observe more subtle signs, the sampling frequency should be in the order of $1200 \mathrm{~Hz}$. This requirement for a higher sampling frequency could be clearly seen in the initial ECG data observed. As a result of this, additional work was carried out to add a second BLE transmission board to the system in order to provide proof-of-concept. This method of sampling frequency increase proved successful, and provides scope to further increase the number of boards (and therefore greater sampling frequency) as required, for more detailed data representation.

\section{EXPERIMENTAL RESULTS}

Experimental testing was carried out to confirm the proofof concept as well as to investigate the best settings for the compression method utilized. Further work is required in order to confirm the universal usage of this design.

The data collected during experimental testing can be found at: http://personal.ee.surrey.ac.uk/Personal/P.Barnaghi/eHealthMonitoring/.

Initial experimentation investigated the latency of the transmitted signal from the sensor platform to the Android display device. As no timing data is currently transmitted alongside the sample value, understanding of the latency of the system is important in reconstructing a valid representation of the measured signals. By definition, the GATT update period should be able to be altered between $7.5 \mathrm{~ms}$ and $4 \mathrm{~s}$, but the current implementation of BLE within the Android operating system means that it is not possible to access this method. As such, the update period is fixed at $10 \mathrm{~ms}$. Experimental results showed a variation in the update period between $9.3 \mathrm{~ms}$ and $10 \mathrm{~ms}$. Further experimentation and measurement of the sensor sampling and retrieval process proved that this observed variation was in fact due wholly to variations in the GATT characteristic updates, as the sensor sampling had a variation of 10us.

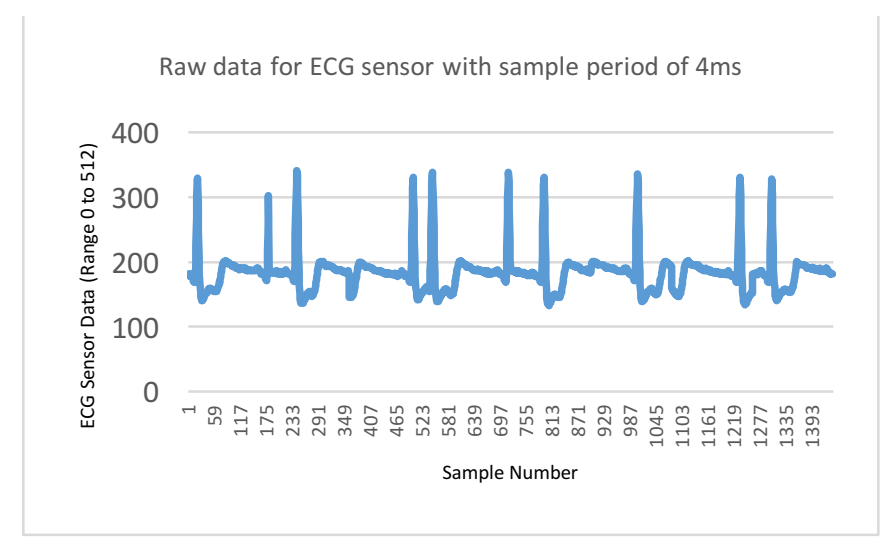

Fig. 3. Raw data for ECG sensor with sample period of $4 \mathrm{~ms}$

Experimentation was also required to find the most suitable segment size and sampling frequency to produce a useable SAX result. Initial ECG measurement at $10 \mathrm{~ms}$ provided a result that allowed for general classification, but did not show the finer details of the signal. The sample period was reduced to $4 \mathrm{~ms}$ and the segment size was then slowly increased until a signal could be decoded. Raw ECG data is shown in Fig. 3.

Fig 4. shows the final result of using a 180 sample segment size with an 8 symbol alphabet, peak limiting and a rolling 5 point average on the raw data shown above. A clear correlation can be seen between the raw data and the final signal. With the processor module used and the current software, there are limitations to the segment size that can be used due to the large amount of memory required to process the data. 


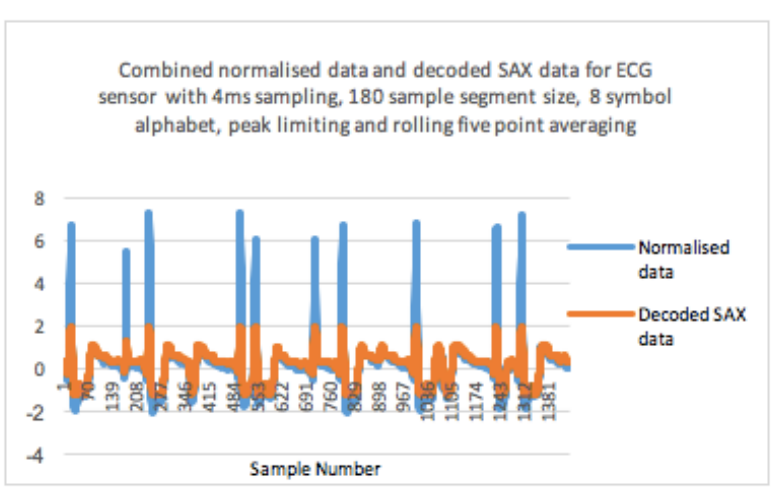

Fig. 4. Combined representaton of normalised and decoded SAX data

\section{CONCLUSIONS}

Research has shown that a limited number of wirelessmonitoring devices exist, but that conversely there is a large requirement for their use within a pediatric environment. It has highlighted the potential pitfalls of such a system, in terms of the need to provide sensors that are sympathetic to the patient, as well as the need for them to work reliably in an environment subject to movement and interference by the patient.

A working prototype has been produced that allows data to be retrieved from four different sensors (SpO2, BPM, Temperature and ECG). This data is wirelessly transmitted via Bluetooth Low Energy to a midpoint where it is stored and can also be displayed. This stored data can then be transmitted via email in the form of a comma separated document to a recipient of the users' choice. The user has full control over the unit connectivity, sampling period, and collected data, as well as the implementation of compression methods if required.

The temperature sensor has two types of software compression implemented as options for the user; in the form of PAA and adaptive compression. The ECG sensor also has two types of software compression; comparative measurement and SAX compression. The sampling frequency for the ECG sensor is above the capabilities of the BLE standard to transmit, and therefore a form of parallel transmission via two BLE boards has been implemented in order to increase the sampling frequency if the user withes to avoid data compression.

The completion of the working prototype has proven that the principle of a low cost device using readily available components is completely feasible, but also that work is required to advance the sensor technology to make it more likely that children will not interfere or remove the sensors.

\section{ACKNOWLEDGMENT}

The authors would like to thank Prof. Roma Maguire for suggesting this project and for assistance provided throughout.

\section{REFERENCES}

[1] Hospital Episode Statistics Analysis, Health and Social Care Information Centre, Hospital Episode Statistics: Admitted Patient Care, England 2014-15, 25 November 2015.
[2] IDC Press Release Nov 2014, Available at: http://www.idc.com/getdoc.jsp?containerId=prUS25262514, Last accessed in April 2016.

[3] Cooking Hacks eHealth Sensor Shield, Available at: https://www.cooking-hacks.com/documentation/tutorials/ehealthbiometric-sensor-platform-arduino-raspberry-pi-medical, Last accessed in April 2016.

[4] Arduino Mega 2560, Available at: https:/www.arduino.cc/en/Main/arduinoBoardMega2560, Last accessed in April 2016

[5] Adafruit Bluetooth LE shield, Available at: https://www.adafruit.com/products/2746, Last accessed in April 2016

[6] Jones,V., Bults, R., Konstantas, D., and Vierhout, P., "Healthcare PANs: Personal Area Networks for trauma care and home care," Fourth International Symposium on Wireless Personal Multimedia Communications (WPMC), Aalborg, Denmark, 2001

[7] Konstantas, D., Jones, V., and Herzog, R., "MobiHealth-Innovative 2.5/3G mobile services and applications for health care," The IST Mobile \& wireless telecommunications Summit 2002 Thessaloniki. Greece, 16-19 Jun 2002

[8] Konstantas, D., van Halteren, A., Bults, R., Wac, K., Widya, I., Jones, V., Dokovsky, N., Koprinkov, G., and Herzog, R., "Mobile patient monitoring: the MobiHealth System," International Congress on Medical and Care Compunetics (NCC04), 2-4 June 2004, Den Haag, Netherlands. pp. 25-32

[9] Lo, Benny PL, Thiemjarus, S., King, R., and Guang-Zhong Yang, "Body sensor network - A wireless sensor platform for pervasive healthcare monitoring," unpublished, 2005.

[10] Otto, C., Milenković, A., Sanders, C., Jovanov, E., "System architecture of a wireless body area sensor network for ubiquitous health monitoring," Journal of mobile multimedia 1.4 (2006): 307-326.

[11] TinyOS, Available at: https://github.com/tinyos/tinyos-main/, Last accessed in July 2016

[12] Milenković, A., Otto, C., and Jovanov, E., "Wireless sensor networks for personal health monitoring: Issues and an implementation," Computer communications 29.13 (2006): 2521-2533.

[13] Abidoye, A., Azeez, N., Adesina, A., Agbele, K., and Nyongea, H., "Using wearable sensors for remote healthcare monitoring system," Journal of Sensor Technology, 2011, 1:22-28

[14] Snehalatha, D., and Shruti, M., "Design of a healthcare monitoring system using wearable and environmental sensor," International Journal of Engineering Research and General Science Volume 3, Issue 2, Part 2, March-April 2015

[15] SHIMMER, Available at: http://www.shimmersensing.com, Last visited in April 2016

[16] Burns, A., Greene, B., McGrath, M., O’Shea, T., Kuris, B., Ayer, S., Stroiescu, F., and Cionca, V., "SHIMMER'M - A wireless sensor platform for noninvasive biomedical research," IEEE Sensors Journal, Vol. 10, No.9, September 2010

[17] Fortino, G., Parisi, D., Pirrone, V., and Di Fatta, G., "BodyCloud: A SaaS approach for community body sensor networks," Future Generation Computer Systems 35 2014: 62-79

[18] Berg, K., "Heart-rate telemetry for evaluation of the energy expenditure of children with cerebral palsy," The American Journal of Clinical Nutrition 23:December 1971, pp. 1438-1445

[19] Gellermann, J., Kraft, S., and Ehrich, J., "Twenty-four-hour ambulatory blood pressure monitoring in young children," Pediatric Nephrology, 1997 11:707-710.

[20] Salgado, C., Jardim, P., Viana, J., Jardim, T., and Velasquez, "Home blood pressure in children and adolescents: a comparison with office and ambulatory blood pressure measurements," Acta Paediatrica 2011, 100: e163-e168

[21] Saarel, E., Doratotaj, S. and Sterba, R, "Initial experience with novel mobile cardiac outpatient telemetry for children and adolescents with suspected arrhythmia," Congenital Heart Disease 2008, 3: 33-38

[22] CardioNet, Available at: https://www.cardionet.com/, Last visited in April 2016 
[23] LifeTouch, Isansys, Available at: http://www.isansys.com, Last visited in April 2016

[24] WristOx, Nonin, Available at: http://www.nonin.com, Last visited April 2016.

[25] Cameron, S., "Piece-wise linear approximations," DTIC Document, Tech. Rep., 1966.

[26] Keogh, E., Chakrabarti, K., Pazzani, M., and Mehrotra,

S., "Dimensionality reduction for fast similarity search in large time series databases," Knowledge and Information Systems 3(3): 263-286. 2000

[27] Sant'Anna, A., and Wickström, N., "Symbolization of time-series: An evaluation of SAX, PERSIST, and ACA," Image and Signal Processing (CISP), 2011 4th International Congress on. Vol. 4. IEEE, 2011.

[28] Lin, J., Keogh, E., Lonardi, S., and Chiu, B., "A symbolic representation of time series, with implications for streaming algorithms," Proceedings of the 8th ACM SIGMOD workshop on Research issues in data mining and knowledge discovery (pp. 2-11). ACM. June 2003.

[29] Kulahcioglu, B., Ozdemir, S., and Kumova, B., "Application of symbolic Piecewise Aggregate Approximation (PAA) analysis to ECG signals," 17th IASTED International Conference on Applied Simulation and Modelling. 2008.

[30] Singh, B., Kaur, A., and Singh, J., "A review of ECG data compression techniques," International Journal of Computer Applications 116.11 2015.

[31] Gomez, C., Joaquim O., and Paradells, J., Overview and evaluation of bluetooth low energy: An emerging low-power wireless technology. Sensors 12.9 (2012): 11734-11753

[32] Dickinson, D., The normal ECG in childhood and adolescence. Heart. 2005;91(12):1626-1630.

[33] Rijnbeek, P., Kors, J. and Witsenburg, M., Minimum Bandwidth Requirements for Recording of Pediatric Electrocardiograms. Circulation. 2001; 104:3087-3090.

[34] Rijnbeek, P., Witsenburg, M., Schrama, E., Hess, J., and Kors, J., New normal limits for the paediatric electrocardiogram, European Heart Journal (2001) 22, 702-711. 